\title{
TENSOR PRODUCT OF HOPF BIMODULES OVER A GROUP
}

\author{
CLAUDE CIBILS \\ (Communicated by Ken Goodearl)
}

\begin{abstract}
We describe the monoidal structure of the category of Hopf bimodules of a finite group and we derive a surjective ring map from the Grothendieck ring of the category of Hopf bimodules to the center of the integral group ring. We consider analogous results for the multiplicative structure of the Hochschild cohomology.
\end{abstract}

\section{INTRODUCTION}

Hopf bimodules are natural representations of a Hopf algebra $H$. They form an abelian category $\mathcal{B}(H)$ provided with a tensor product; their definition is given below. M. Rosso proved in [16] that $\mathcal{B}(H)$ is equivalent - as a monoidal category - to the category of modules over the Drinfeld double of $H$; therefore $\mathcal{B}(H)$ is a braided category (see $[2,7,9,11])$. M. Rosso and S.L. Woronowicz $([15,18])$ have considered those structures in relation to differential calculus on quantum groups; Hopf bimodules over a group algebra provides the classification of quantum structures on path algebras obtained in [4].

The main purpose of this paper is to describe the tensor product of Hopf bimodules over an arbitrary group $G$ with coefficients in a ring $k$, which are $k$-projective and finitely generated. Alternatively, if $G$ is finite the result concerns Hopf bimodules without restrictions on their $k$-structure. Notice that the computation of the monoidal structure of Hopf bimodules is perhaps related with the work of Wasserman concerning the computation of Conne's fusion for $S U(2)$, see [17] and [10].

The quoted structure result is performed by constructing an explicit monoidal category in terms of conjugacy classes and representations of the centralizers provided with a tensor product defined in terms of induced modules. We prove that the resulting category is equivalent - as a monoidal category - to the one formed by the Hopf bimodules. As a consequence, we obtain that the Grothendieck ring of Hopf bimodules surjects on the center of the integral group ring.

These results are related with the study of the Hochschild cohomology algebra of a group algebra. Indeed, we recall that the algebra structure of the latter is unknown and that a link between the tensor product of Hopf bimodules and the cup product of Hochschild cocycles seems to exist; in the abelian group case, such a relation is clear since both structures are graduations over the group of products

Received by the editors July 27, 1995 and, in revised form, December 1, 1995.

1991 Mathematics Subject Classification. Primary 18D10, 20G05, 20G10.

Supported by University of Bern and University of Grenoble.

(C)1997 American Mathematical Society 
of more usual structures (respectively left modules and usual cocycles), see [5]. We consider this point at the last section.

We know from [4] that the category of $k G$-Hopf bimodules is equivalent - just as an abelian category - to the product of categories of usual modules $\prod_{C \in \mathcal{C}} \bmod k Z_{C}$ where $\mathcal{C}$ is the set of conjugacy classes and $Z_{C}$ is the centralizer of one of the elements of $C \in \mathcal{C}$. Objects of this product are possibly infinite families of right modules over the centralizers. W.D. Nichols ([14]) has initiated this classification for a finite abelian group and a field of characteristic not dividing the order of G. D. Dijkgraaf, V. Pasquier and P. Roche in [8] considered the case of a finite group with complex coefficients and modules over the Drinfeld double of the group algebra, using Lusztig's results ([12]) for studying its semisimplicity.

We begin this paper with an account of Hopf bimodules and their monoidal structure, including the proof of the additive structure result obtained in [4] presented here in a simplified version. This enables us to obtain the complete multiplicative structure Theorem in section 3 .

\section{Hopf BIMOdules}

A Hopf bimodule $B$ over a Hopf algebra $H$ is an $H$-bimodule which is simultaneously an $H$-bicomodule; moreover, it is required that the structure maps $\delta_{L}: B \rightarrow H \otimes B$ and $\delta_{R}: B \rightarrow B \otimes H$ are $H$-bimodule maps for the $H$-bimodule structure of the tensor products obtained through the comultiplication of $H$. For more details concerning this definition, see for instance [5].

The tensor product of Hopf bimodules $B$ and $B^{\prime}$ is the bimodule $B \otimes_{H} B^{\prime}$ equipped with the bicomodule structure given by the maps

$$
\begin{aligned}
& B \otimes_{H} B^{\prime} \stackrel{\delta_{L} \otimes \delta_{L}}{\longrightarrow}(H \otimes B) \otimes_{H}\left(H \otimes B^{\prime}\right) \stackrel{1 \otimes \tau \otimes 1}{\longrightarrow} H \otimes_{H} H \otimes B \otimes_{H} B^{\prime} \stackrel{m \otimes 1 \otimes 1}{\longrightarrow} H \otimes B \otimes_{H} B^{\prime}, \\
& B \otimes_{H} B^{\prime} \stackrel{\delta_{R} \otimes \delta_{R}}{\longrightarrow}(B \otimes H) \otimes_{H}\left(B^{\prime} \otimes H\right) \stackrel{1 \otimes \tau \otimes 1}{\longrightarrow} B \otimes_{H} B^{\prime} \otimes H \otimes_{H} H \stackrel{1 \otimes 1 \otimes m}{\longrightarrow} B \otimes_{H} B^{\prime} \otimes H,
\end{aligned}
$$

where $\tau$ is the flip map and $m$ is the multiplication map of $H$. There is no difficulty in checking that $1 \otimes \tau \otimes 1$ is a well defined $H$-bimodule map; both compositions give $H$-bimodule maps and define an Hopf bimodule structure on the bimodule $B \otimes_{H} B^{\prime}$.

Theorem 2.1 ([4]). Let $k$ be a ring and $G$ be a group. The category $\mathcal{B}(k G)$ of $k G$-Hopf bimodules is equivalent to $\prod_{C \in \mathcal{C}} \bmod k Z_{C}$.

Proof. Let $M=\left(M_{C}\right)_{C \in \mathcal{C}}$ be a possibly infinite family of right $k Z_{u(C)}$-modules, where $Z_{u(C)}$ is the centralizer of a chosen element $u(C)$ in each conjugacy class $C$. Let $V M$ be the $k G$-bimodule $\prod_{C \in \mathcal{C}} k G \otimes M_{C} \uparrow_{Z_{C}}^{G}$ where the right structure of $M_{C} \uparrow_{Z_{C}}^{G}$ is the induced one - recall that $M_{C} \uparrow_{Z_{C}}^{G}=M_{C} \otimes_{k Z_{C}} k G$ - and the left structure is the trivial one. The $k$-module $k G$ is provided with the regular left and right actions and each tensor product is equipped with the resulting diagonal left and right actions.

Concerning the bicomodule structure, it is useful to know (see [13]) that any Hopf bimodule is bigraded by $G$ : we have $B=\bigoplus_{x, y \in G}{ }^{y} B^{x}$ where ${ }^{y} B^{x}$ is the isotypic cocomponent of type $(y, x)$, i.e.

$$
{ }^{y} B^{x}=\left\{b \in B \mid \delta_{L}(b)=y \otimes b \text { and } \quad \delta_{R}(b)=b \otimes x\right\} .
$$


Notice that if $G$ is finite $(k G)^{*}$ is a product of copies of $k$ provided by the complete set of orthogonal idempotents given by the Dirac maps; results about $k G$ bicomodules are duals of results about $(k G)^{*}$-bimodules and the latter ones are $G$-bigraded by the isotypic components.

The isotypic cocomponents of $V M$ are defined as follows:

$$
x \otimes M_{C} \otimes K={ }^{x K^{-1} u(C) K}(V M)^{x}
$$

where $K$ is a left class of $Z_{u(C)} \backslash G$. It is easily checked that this data provides $V M$ with a Hopf bimodule structure.

The reverse functor $W$ associates to a Hopf bimodule $B$ the collection of isotypic cocomponents $\left\{{ }^{u(C)} B^{1}\right\}_{C \in \mathcal{C}}$. Notice that ${ }^{u(C)} B^{1}$ is a right $k Z_{u(C)}$-module through "conjugation" of actions: $b . z=z^{-1} b z$. Indeed, the right action takes ${ }^{u(C)} B^{1}$ to ${ }^{u(C) z} B^{z}$ and the left action returns it to ${ }^{z^{-1} u(C) z} B^{1}$. Since $z \in Z_{u(C)}$, the latter is exactly ${ }^{u(C)} B^{1}$ as required.

Remark 2.2. Let $\mathcal{B}_{k}(k G)$ be the full subcategory of $\mathcal{B}(k G)$ of Hopf bimodules with underlying $k$-module projective and finitely generated, and let $\times_{C \in \mathcal{C}} \bmod { }_{k} k Z_{C}$ be the category of finite families of right $k Z_{C}$-modules which are $k$-projective and finitely generated. The functors of the preceding proof restrict to equivalences between these sub-categories.

\section{Monoidal struCture}

We define first the monoidal category $\mathcal{M}(k G)$ mentioned in the introduction for $k$ a commutative ring and $G$ any group. Objects are formal finite sums $\sum_{C \in \mathcal{C}}\left[M_{C}\right] C$ where $M_{C}$ is a right module over the centralizer $Z_{u(C)}$ of a chosen element $u(C)$ of each conjugacy class $C$, such that the underlying $k$-structure of $M_{C}$ is projective and finitely generated. Morphisms and direct sums are the natural ones.

In order to define the monoidal structure of $\mathcal{M}(k G)$, recall that $M \uparrow_{Z}^{G}$ is the induced module $M \otimes_{k Z} k G$ of a right $k Z$-module $M$ where $Z$ is a sub-group of a group $G$. The tensor product is defined on elementary objects,

$$
[M] A \otimes[N] B=\sum_{C \in \mathcal{C}}\left[X_{A, B}^{C}\right] C,
$$

where $X_{A, B}^{C}$ is a sub $k Z_{u(C)}$-module of $\left(M \uparrow_{Z_{u(A)}}^{G} \otimes N \uparrow_{Z_{u(B)}}^{G}\right) \downarrow_{Z_{u(C)}}$. Letting

$$
E_{A, B}^{C}=\left\{(K, L) \in Z_{u(A)} \backslash G \times Z_{u(B)} \backslash G \mid K^{-1} u(A) K L^{-1} u(B) L=u(C)\right\},
$$

we define

$$
X_{A, B}^{C}=\bigoplus_{(K, L) \in E_{A, B}^{C}} M \otimes K \otimes_{k} N \otimes L .
$$

Notice that $E_{A, B}^{C}$ is actually preserved by right multiplication by elements of $G$. We are able to extend this product to the full category since objects are finite formal sums. It is not clear a priori that this product is associative; once the next Theorem is proved the associativity will follow since the tensor product of Hopf bimodules is clearly associative.

Theorem 3.1. Let $k$ be a ring and $G$ be any group. The categories $\mathcal{B}_{k}(k G)$ and $\mathcal{M}(k G)$ are equivalent through functors which preserve tensor products. 
Proof. We refer to the proof of the previous Theorem 2.1 for notations and definitions. Let $I$ and $I^{\prime}$ be Hopf bimodules constructed via the functor $V$ by means of elementary objects $[M] A$ and $[N] B$, and consider their tensor product $I \otimes_{k G} I^{\prime}=$ $V([M] A) \otimes_{k G} V([N] B)$. We need to prove that $W$ of this tensor product is the announced object of $\mathcal{B}_{k}(k G)$; in other words we have to show that

$$
{ }^{u(C)}\left(I \otimes_{k G} I^{\prime}\right)^{1}=X_{A, B}^{C}
$$

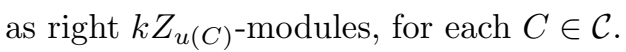

First we collect the following easy equality:

$$
{ }^{d} I^{c} \otimes_{k G}{ }^{x a} I^{\prime x b}={ }^{d c} I^{c x} \otimes_{k G}{ }^{a} I^{\prime b} \subset{ }^{d x a}\left(I \otimes_{k G} I^{\prime}\right)^{c x b} .
$$

In particular this means that a normalization can be performed as follows:

$$
I \otimes_{k G} I^{\prime}=\bigoplus_{z, y, x \in G}{ }^{z} I^{y} \otimes_{k}{ }^{x} I^{\prime 1}
$$

and the isotypic cocomponent ${ }^{v}\left(I \otimes_{k G} I^{\prime}\right)^{u}$ is given in this normalization by $\bigoplus_{y x=v}{ }^{y} I^{u} \otimes_{k}{ }^{x} I^{\prime 1}$. Hence

$$
{ }^{u(C)}\left(I \otimes_{k G} I^{\prime}\right)^{1}=\bigoplus_{x y=u(C)}{ }^{y} I^{1} \otimes_{k}{ }^{x} I^{\prime 1} .
$$

Now we have $I=V([M] A)=k G \otimes_{k} M \uparrow_{Z_{u(A)}}^{G}$, and by definition of the isotypic cocomponents

$$
z \otimes M \otimes K={ }^{z K^{-1} u(C) K} I^{z}
$$

for each $z \in G$ and $K \in Z_{u(A)} \backslash G$. Consequently the isotypic cocomponents of type ${ }^{y} I^{1}$ are of the form ${ }^{K^{-1} u(C) K} I^{1}$ for all $K \in Z_{u(A)} \backslash G$ and we derive

$$
\begin{aligned}
{ }^{u(C)}\left(I \otimes_{k G} I^{\prime}\right)^{1}= & \left.\bigoplus_{(K, L) \in E_{A, B}^{C}} K^{-1} u A\right) K^{1} I_{k}{ }^{L^{-1} u(B) L} I^{\prime 1} \\
& =\bigoplus_{(K, L) \in E_{A, B}^{C}}(1 \otimes M \otimes K) \otimes(1 \otimes N \otimes L) .
\end{aligned}
$$

The identification with the described $k$-module $X_{A, B}^{C}$ is now clear. To end the proof it is enough to record that the right action of $Z_{u(C)}$ on this direct sum coincides with the one described on $X_{A, B}^{C}$. Indeed, the action is given by "conjugation" of the actions (cf. the proof of 2.1) and the left structure on the right induced modules is the trivial one.

We consider now the Grothendieck ring $K_{0} \mathcal{B}_{k}(k G)$ of the category of $k$-projective and finitely generated Hopf bimodules over $k G$, namely the free abelian group on the set of isomorphism classes of objects of $\mathcal{B}_{k}(k G)$ divided by the subgroup generated by the relations $Y-Y^{\prime}-Y^{\prime \prime}$ associated to each split exact sequence $0 \rightarrow Y^{\prime} \rightarrow Y \rightarrow Y^{\prime \prime} \rightarrow 0$ of the category. In case the category is a Krull-Schmidt one; this quotient is free with basis the set of isomorphism classes of indecomposable objects. If $k$ is a field, the monoidal structure of the category induces a product on the Grothendieck group which provides a ring structure with unit (notice that the Hopf algebra is the unit object for the tensor product of Hopf bimodules). 
Corollary 3.2. Let $k$ be a field and $G$ a finite group. There is a ring surjection

$$
\phi: K_{0} \mathcal{B}(k G) \longrightarrow \mathcal{Z}(\mathbf{Z} G)
$$

where $\mathcal{Z}(\mathbf{Z} G)$ is the center of the group algebra $\mathbf{Z} G$.

Proof. Recall first that $\mathcal{Z}(\mathbf{Z} G)$ is free with basis $\{\bar{C}\}_{C \in \mathcal{C}}$ where $\bar{C}$ is the sum in $\mathbf{Z} G$ of all the elements of the conjugacy class $C$, see for instance [6]. The structure constants of this ring are non negative integers given by $\bar{A} \bar{B}=\sum_{C \in \mathcal{C}} e_{\mathrm{A}, \mathrm{B}}^{\mathrm{C}} \bar{C}$. It is elementary to prove that $e_{\mathrm{A}, \mathrm{B}}^{\mathrm{C}}$ is the cardinal of the set $E_{A, B}^{C}$ considered before.

Next we identify $K_{0} \mathcal{B}(k G)$ and $K_{0} \mathcal{M}(k G)$ through the functors $V$ and $W$ and we define

$$
\phi\left(\sum_{C \in \mathcal{C}}\left[M_{C}\right] C\right)=\sum_{C \in \mathcal{C}} \operatorname{dim}_{k}\left[M_{C}\right] \bar{C} .
$$

This gives a ring homomorphism since from one side

$$
\begin{aligned}
\phi([M] A \otimes[N] B) & =\phi\left(\sum_{C \in \mathcal{C}}\left[X_{A, B}^{C}\right] C\right)=\sum_{C \in \mathcal{C}} \operatorname{dim}_{k} X_{A, B}^{C} \bar{C} \\
& =\sum_{C \in \mathcal{C}} \operatorname{dim}_{k} m \operatorname{dim}_{k} N\left|E_{A, B}^{C}\right| \bar{C}
\end{aligned}
$$

and from the other side we have

$$
\phi([M] A) \phi([N] B)=\left(\operatorname{dim}_{k} M \bar{A}\right)\left(\operatorname{dim}_{k} N \bar{B}\right)=\operatorname{dim}_{k} M \operatorname{dim}_{k} N \sum_{C \in \mathcal{C}} e_{\mathrm{A}, \mathrm{B}}^{\mathrm{C}} \bar{C} .
$$

Each basis element $\bar{C}$ of $\mathcal{Z}(\mathbf{Z} G)$ is reached through $\phi$, using the elementary object having the trivial representation of the centralizer of $C$ as coefficient of $C$.

Remark 3.3. It would be interesting to know if a suitable version of the preceding surjection still exists from the Grothendieck ring of Hopf bimodules over a finite dimensional Hopf algebra $H$ to the center of an integral form of $H$.

\section{HochSCHILD COHOMOLOGY}

The behavior of the cup product for the Hochschild cohomology classes of a finite group is probably similar to the behavior of the tensor product of Hopf bimodules as described in Theorem 3.1. Indeed, we have at first that the underground additive structures agree through analogous descriptions with respect to centralizers of conjugacy classes, since it is well known that

$$
H H^{*}(k G, k G)=\bigoplus_{C \in \mathcal{C}} H^{*}\left(Z_{C}, k\right)
$$

where $H H^{*}$ denotes Hochschild cohomology and $H^{*}$ usual group cohomology; see for instance [3] or [1] and compare with Theorem 2.1. Moreover in [5] we have obtained that for a finite abelian group the similarity holds since there is an algebra isomorphism between $H H^{*}(k G, k G)$ and $k G \otimes H^{*}(G, k)$, while for Hopf bimodules the category $\mathcal{B}_{k}(k G)$ is equivalent to $[\bmod k G] G$ : for abelian finite groups both multiplicative structures - cup product in Hochschild cohomology and tensor product of Hopf bimodules - are obtained by grading over $G$ simpler multiplicative structures (cup product in usual cohomology and tensor product of usual representations). 
In order to state a precise conjecture for the Hochschild cohomology algebra of a finite group, it is necessary to understand differently the modules $X_{A, B}^{C}$ of the preceding section. I am indebted to J. Thévenaz for pointing out the following

Lemma 4.1. Let $G$ be a finite group and let $k$ be a ring. Let $A$ and $B$ be conjugacy classes and let $a$ and $b$ be chosen elements in $A$ and $B$. Let $M$ and $N$ be right $k Z_{a}$ and $k Z_{b}$-modules, and let $C$ be a conjugacy class with chosen element $c$. The $k Z_{C^{-}}$ module

$$
X_{A, B}^{C}=\bigoplus_{(K, L) \in E_{A, B}^{C}}(M \otimes K) \otimes(N \otimes L) \subset\left(M \uparrow \uparrow_{Z_{a}}^{G} \otimes N \uparrow \uparrow_{Z_{b}}^{G}\right) \downarrow_{Z_{C}}
$$

is isomorphic to

$$
\bigoplus_{E_{A, B}^{C} / Z_{c}}\left(M^{K} \downarrow_{Z_{a}^{K} \cap Z_{b}^{L}} \otimes N^{L} \downarrow_{Z_{a}^{K} \cap Z_{b}^{L}}\right) \uparrow_{Z_{a}^{K} \cap Z_{b}^{L}}^{Z_{c}} .
$$

Recall that $E_{A, B}^{C}=\left\{(K, L) \in Z_{a} \backslash G \times Z_{b} \backslash G \mid K^{-1} a K L^{-1} b L=c\right\}$ and that $Z_{C}$ acts on the right on this set. The latter direct sum is obtained by choosing one element in each orbit of $E_{A, B}^{C}$ under this action. The module $M^{K}$ denotes the same underlying $k$-module as $M$, equipped with the right action of $Z_{a}^{K}=K^{-1} Z_{a} K=$ $Z_{K^{-1} a K}$ defined by $\left(K^{-1} a K\right) M=a m$.

Proof. The module $X_{A, B}^{C}$ decomposes along the orbits of the action of $Z_{C}$ on $E_{A, B}^{C}$. Each resulting piece corresponds to the criteria of being an induced module - see for instance [6] - since its direct summands are transitively permuted by $Z_{C}$ and the stabilizer of $(K, L)$ is $Z_{a}^{K} \cap Z_{b}^{L}$.

We construct now an algebra based on the usual cohomology algebras of the centralizers of conjugacy classes in a way very similar to how we have defined the category $\mathcal{M}(k G)$.

Let $\mathbf{M}(k G)=\bigoplus_{C \in \mathcal{C}} H^{*}\left(Z_{u(C)}, k\right) C$; that is, $\mathbf{M}(k G)$ is the $k$-module of formal linear combinations of conjugacy classes with coefficients in the respective cohomology group (recall that $u$ is a choice of an element in each conjugacy class). Let $m A$ and $n B$ be elementary elements in $\mathbf{M}(k G)$, with $m \in H^{*}\left(Z_{a}, k\right)$ and $n \in H^{*}\left(Z_{b}, k\right)$. The product $m A \cdot n B=\sum_{C \in \mathcal{C}} x_{\mathrm{A}, \mathrm{B}}^{\mathrm{C}} C$ is defined by

$$
x_{\mathrm{A}, \mathrm{B}}^{\mathrm{C}}=\sum_{E_{A, B}^{C} / Z_{c}} \operatorname{Ind}_{Z_{a}^{K} \cap Z_{b}^{L}}^{Z_{c}}\left(\operatorname{Res}_{Z_{a}^{K} \cap Z_{b}^{L}}^{Z_{K}^{K}} m^{K} \smile \operatorname{Res}_{Z_{a}^{L} \cap Z_{b}^{L}}^{Z_{L}^{L}} n^{L}\right)
$$

where $m^{K}$ and $n^{L}$ denotes elements corresponding to $m$ and $n$ in $H^{*}\left(Z_{a}^{K}, k\right)$ and $H^{*}\left(Z_{b}^{L}, k\right)$ obtained through conjugation, Ind and Res are the induction and restriction maps defined in cohomology, and $\smile$ is the cup product in the corresponding cohomology algebra.

Conjecture. The Hochschild cohomology algebra $H H^{*}(k G, k G)$ is isomorphic to $\mathbf{M}(k G)$.

\section{REFERENCES}

[1] Benson, D.J.: Representations and cohomology I: Basic representation theory of finite groups and associative algebras. Cambridge University Press. 1991 MR 92m:20005

[2] Bruguières A., Théorie tannakienne non commutative. Comm.in Algebra 22, 5817-5860 (1994) MR 95m:16028

[3] Cartan, H., Eilenberg, S.:Homological algebra. Oxford University Press. 1956 MR 17:1040e 
[4] Cibils C., Rosso M.: Algèbres des chemins quantiques. Publication interne, Genève (1993) et prépublication de l'IRMA 047, Strasbourg (1993). To appear in Advances in Maths.

[5] Cibils, C., Solotar, A.: Hochschild cohomology algebra and Hopf bimodules of an abelian group. Prepublication.

[6] Curtis, C.W., Reiner, I.: Methods of representation theory, Pure \& applied mathematics, Wiley-interscience, New York, 1981 MR 90k:20001

[7] Deligne, P. : Catégories tannakiennes. Progress in Mathematics. The Grothendieck Festschrift. Vol II, pp 110-195. Birkhäuser 1990 MR 92d:14002

[8] Dijkgraaf, D. Pasquier,V. Roche, P.: Quasi Hopf algebras, group cohomology and orbifold models. Nuclear Physics B (Proc. Suppl.) 18B 60-72, (1990) MR 92m:81238

[9] Drinfeld, V.G.: Quantum groups. Proceedings of the International Congress of Mathematicians. Vol. 1, pp. 798-820. Berkeley: Academic Press 1986 MR 89f:17017

[10] Jones, V.: Fusion en algèbres de von Neumann et groupes de lacets [d'après A. Wassermann]. Séminaire Bourbaki 8001995

[11] Kassel, C.: Quantum groups. Grad. Texts in Math. 1551995 MR 96e:17041

[12] Luzstig, G. Leading coefficients of character values of Hecke algebras. Arcata Conference on Representations of Finite Groups. Proc. of Symp. in Pure Math. 47 A.M.S. 1987

[13] Montgomery, S.: Hopf algebras and their actions on rings, CBMS 82, Amer. Math. Soc., 1982 MR 94i: 16019

[14] Nichols, W.D.: Bialgebras of type one. Commun. Algebra 6, 1521-1552 (1978) MR 58:22150

[15] Rosso, M.: Algèbres enveloppantes quantifiées, groupes quantiques compacts de matrices et calcul différentiel non commutatif. Duke Math. J. 61, 11-40 (1978) MR 91i:17022

[16] Rosso, M.: Groupes quantiques et algèbres de battages quantiques, C.R. Acad. Sci. Paris 320, 145-148 (1995) MR 95m:17015

[17] Wassermann, A.: Fusion for von Neumann algebras and loop groups, to appear.

[18] Woronowicz, S.L.: Differential calculus on compact matrix pseudogroups (quantum groups). Commun. Math. Phys. 122, 125-170 (1989) MR 90g:58010

Mathematisches Institut, Universität Bern, Sidlerstrasse 5, CH-3012 Bern, SwitzerLAND

Institut Fourier, Laboratoire de Mathématiques, URA 188 du CNRS, BP 74, F-38402 St. Martin d'Hères cedex, France

E-mail address: cibils@math.univ-montp2.fr

Current address: Départemente de Mathématiques, Université de Montpellier 2, F-34095

Montpellier cedex 5, France 